\title{
Aplicaciones y desarrollo de prototipos con Internet de las Cosas
}

\section{Applications and development of prototypes with Internet of Things}

\author{
ROMO-GONZALEZ, Ana Eugenia†* \& VILLALOBOS-ALONZO, María de los Ángeles \\ Universidad Tecnológica de Jalisco, Tecnologías de la Información. Desarrollo de Software multiplataforma \\ Universidad Tecnológica de Jalisco, Mantenimiento Industrial
}

ID $1^{\text {er }}$ Autor: Ana Eugenia, Romo-González / ORC ID: 0000-0002-4653-2593, CVU -212291

ID $1^{\mathrm{er}}$ Coautor: María de los Ángeles, Villalobos-Alonzo / ORC ID: 0000-0003-3052-8271, CVU CONACYT ID: 212718

DOI: $10.35429 / J O C T .2019 .9 .3 .1 .7$

Recibido 03 de Enero, 2019; Aceptado 15 Marzo, 2019

\section{Resumen}

En el paradigma de Internet de las Cosas (IoT) se considera que los múltiples objetos interconectados en la red pueden interactuar y cooperar entre sí para proporcionar servicios y generar aplicaciones nuevas. La construcción de estos entornos inteligentes proporciona oportunidades de mejoras económicas y sociales en los países que trae consigo nuevos retos para las industrias de desarrollo de hardware y software y para el área académica. En este contexto, proporcionar el conjunto de componentes que constituyen la estructura para el desarrollo de prototipos y sus aplicaciones en el área de IoT es el objetivo del presente documento. La metodología empleada es cualitativa de alcance descriptivo debido a que se identifican, clasifican y presentan las características básicas para la definición de proyectos integrales en IoT. La esquematización presentada constituye un marco de referencia que contribuye a la capacitación de capital humano especializado en el área tecnológica al brindar un método formal para la sistematización de aplicaciones que puedan controlar objetos conectados a través de Internet.

Construcción de aplicaciones, Prototipos, Internet de las Cosas

\begin{abstract}
In the Internet of Things paradigm (IoT) it is considered that the multiple interconnected objects in the network can interact and cooperate with each other to provide services and generate new applications. The construction of these intelligent environments provides opportunities for economic and social improvement in the countries that brings new challenges for the hardware and software development industries and for the academic area. In this context, providing the set of components that constitute the structure for the development of prototypes and their applications in the IoT area is the objective of this document. The methodology used is qualitative with a descriptive scope because it identifies, classify and present the basic characteristics for the definition of integral IoT projects. The presented schematization constitutes a frame of reference that contributes to the training of human capital specialized in the technological area by providing a formal method for the systematization of applications that can control objects connected through the Internet.
\end{abstract}

Construction of applications, Prototypes, Internet of Things

Citación: ROMO-GONZALEZ, Ana Eugenia \& VILLALOBOS-ALONZO, María de los Ángeles. Aplicaciones y desarrollo de prototipos con Internet de las Cosas. Revista de Tecnologías Computacionales. 2019 3-9: 1-7

\footnotetext{
* Correspondencia del Autor: (Correo electrónico: aromo@utj.edu.mx)

$\uparrow$ Investigador contribuyendo como primer autor.
} 


\section{Introducción}

La relevancia de Internet de las Cosas (IoT) como concepto y paradigma se sustenta en la existencia de 30 mil millones de dispositivos inteligentes conectados a Internet, proyección al año 2020 (Statista, 2018) que septuplicará la cantidad de personas en el planeta.

Esta característica de súper conectividad que le permite a los diversos tipos de dispositivos y sensores transmitir datos desde cualquier lugar se debe, principalmente, a la malla satelital de redes digitales que rodea el mundo y al uso generalizado de las tecnologías de conexión inalámbrica.

Además, la identificación por radiofrecuencia (RFID) y las tecnologías de red de sensores junto con los sistemas y las tecnologías de la información se encuentran integrados en el entorno (Gubbi, Buyya, Marusic \& Palaniswami, 2013), misma que incluso puede ser imperceptible para los usuarios.

La infraestructura virtual que permite integrar todos los sistemas, dispositivos y servicios, es el cómputo en la nube, ya que los cambios en el entorno físico pueden realizarse a través de software especializado sin la intervención de persona alguna.

La necesidad de plataformas y sistemas especializados para IoT se debe a que la transmisión masiva de datos no provee ventajas sociales o económicas por sí misma, por lo que el análisis de las enormes cantidades de información que se genera por la supervisión y evaluación de los múltiples objetos conectados a Internet representa un reto mayúsculo.

Sin embargo, el reto del análisis masivo de datos se ha vuelto impostergable por las posibilidades que representa para la resolución de problemas de carácter prioritario, como los relacionados con la salud o el medio ambiente.

También, la masificación de datos derivada de la interacción directa entre empresas y usuarios, ha logrado acelerar los beneficios económicos en las cadenas productivas (Evans, 2011) eliminando a los intermediarios y disminuyendo los costos para los clientes, haciendo evidente para la población la utilidad de la digitalización.
En estos entornos, la conectividad se vuelve inteligente ya que los programas analizan los datos de millones de sensores modificando su estructura y haciéndola sensible al contexto.

Las reglas y las restricciones con las que operan las redes pueden delimitarse claramente con el objetivo de que el sistema pueda reconfigurarse de manera automática, a este tipo de redes se les denominan basadas en reglas intención, IBN o Intent Based Networking (Tsuzaki \& Okabe, 2017; Rojas, 2017).

Es en este vórtice de súper convergencia tecnológica en donde surgen oportunidades para el desarrollo de aplicaciones en los ámbitos gubernamentales, empresariales, sociales y personales, y es aquí que surge la pertinencia del presente proyecto.

En el transcurso de la última década se ha vuelto factible la aplicación del Internet de las Cosas para resolver las problemáticas que a nivel internacional presentan las Megapolis.

Dado que las problemáticas de las grandes ciudades refieren al uso eficiente de la energía, del agua, la disposición de residuos orgánicos e inorgánicos, el control de la calidad del aire, la seguridad alimentaria, la generación de empleos, la manufactura y la producción en masa así como la optimización del transporte y de sus vías; este trabajo es pertinente y establecer la plataforma y las especificaciones técnicas para el desarrollo de aplicaciones y prototipos de IoT es el objetivo de este trabajo.

Para el desarrollo de la investigación se identifican y clasifican los elementos fundamentales con base en la literatura en la que se presentan desarrollos de proyectos en el área de IoT, por lo que la metodología utilizada es cualitativa con alcance descriptivo de la que se desprende una esquematización presentada en el apartado de conclusiones.

Los fundamentos para esquematizar proyectos de Internet de las cosas se muestran en los siguientes tres apartados que son previos a la definición de la metodología a desarrollar para el contraste de resultados y de las conclusiones finales. 


\section{Modelo de referencia en IoT}

Los modelos para la construcción de proyectos en IoT se basan en una estructura por niveles, denominada arquitectura en la que se especifican los elementos que componen cada capa y su esquema de interoperatividad.

Sin embargo, debido a la diversidad de dispositivos, protocolos y aplicaciones existentes las estructuras propuestas se enfocan en cumplir una serie de requisitos funcionales necesarios es estos esquemas de sistemas embebidos como la integración de los elementos de la red y la garantía de escalabilidad. Entre las arquitecturas o modelos de referencia propuestos por asociaciones, investigadores y empresas destacan:

1. El proyecto de investigación Europeo FP7

2. ITU

3. ARM del Foro Internacional de IoT

4. IoT de IMB

5. IoT de Intel

6. Microsoft Azure

7. Pragma (Web services - Amazon)

Aunque existen otras arquitecturas, en cada propuesta se debe establecer una manera de estandarizar el acceso a los dispositivos y de alcanzar la máxima integración de elementos para volver transparente la complejidad de su estructura.

El objetivo central consiste en garantizar la fluidez de la gran cantidad de datos que se analiza para posteriormente ser presentada como información válida al usuario final de las aplicaciones que provee el sistema.

La estructura que presentan las múltiples arquitecturas obedece a un estudio internacional realizado por el proyecto IOT-A ${ }^{2}$ (Vermesan et al, 2011) en el que se definen al menos cinco niveles operativos llamados: funcional, de dominio, de comunicación, de información y de seguridad. Una de las arquitecturas más difundida para el desarrollo de proyectos en IoT se conoce como el modelo de referencia de 7 niveles o capas promovido por el Foro mundial de IoT (Bradley, Loucks, Macaulay, \& Noronha, 2014), la tabla 1 muestra la arquitectura por nombre y elementos que lo constituyen.

\begin{tabular}{|l|l|lr|}
\hline Nivel & \multicolumn{1}{|c|}{ Nombre } & \multicolumn{2}{|c|}{ Elementos } \\
\hline 7 & $\begin{array}{l}\text { Colaboración y } \\
\text { procesos }\end{array}$ & $\begin{array}{l}\text { Procesos de negocio e } \\
\text { involucramiento } \\
\text { personas de }\end{array}$ \\
\hline 6 & Aplicación & $\begin{array}{l}\text { Reportes, analítica y } \\
\text { control }\end{array}$ \\
\hline 5 & $\begin{array}{l}\text { Abstracción de } \\
\text { datos }\end{array}$ & Agregaciones y accesos \\
\hline 3 & $\begin{array}{l}\text { Acumulación de } \\
\text { datos }\end{array}$ & $\begin{array}{l}\text { Almacenamiento } \\
\text { la fomputación en }\end{array}$ & $\begin{array}{l}\text { Elementos de análisis de } \\
\text { datos y transformación }\end{array}$ \\
\hline 2 & $\begin{array}{l}\text { Conectividad } \\
\text { dromunicaciones } \\
\text { unidades } \\
\text { procesamiento }\end{array}$ \\
\hline 1 & $\begin{array}{l}\text { Dispositivos } \\
\text { físicos } \\
\text { controladores y }\end{array}$ & $\begin{array}{l}\text { Sensores, actuadores, } \\
\text { dispositivos, máquinas } \\
\text { inteligentes, nodos de } \\
\text { todos tipos }\end{array}$ \\
\hline
\end{tabular}

Tabla 1 Esquema general del modelo de referencia de IoT propuesto por el Foro mundial

Fuente: Elaboración basada en (Bradley et al., 2014)

La definición de cada dispositivo, elementos y protocolo en las capas que lo constituyen depende la implementación, por lo que los estándares propuestos se presentan en el siguiente apartado.

Además de la relación presentada en la Tabla 1, en los modelos de referencia se identifican tres aspectos primordiales que deben incluirse al momento de implementar los proyectos de IoT:

1. Gestionar los dispositivos y los datos asociados.

2. Utilizar elementos de conectividad y de comunicación

3. Realizar procesos de Analítica y diseño de las aplicaciones.

Incluir los incisos del 1 al 3 es un requisito indispensable y también debe existir un mecanismo de integración se permita coordinar y centralizar los datos para su posterior análisis.

\section{Protocolos vinculados al Modelo de referencia en IoT}

Para definir los estándares de comunicación que se utilizan en los niveles o capas de la Tabla 1 se debe considerar que los dispositivos conectados a la red que se agregan en el nivel 1 (o capa de las "cosas") pueden ser de bajo costo y estar limitados, por lo que suelen utilizar una razón baja de tasas de transmisión de datos, como la propuesta mostrada en la Tabla 2. 


\begin{tabular}{|l|l|l|}
\hline \multicolumn{2}{|c|}{ Nivel } & \multicolumn{2}{c|}{ Servicio / estándar } \\
\hline 7 & Servicios web \\
\hline 6 & REST & SOAP \\
\hline 5 & HTTP, MQTT & CoAp \\
\hline 4 & TCP & UDP \\
\hline 3 & IPv6 \\
\hline 2 & 6loWPAN \\
\hline 1 & IEEE 802.15 .4 \\
\hline
\end{tabular}

Tabla 2 Relación de niveles con estándares propuestos para el modelo de referencia de IoT

Fuente: Elaboración basada en (González, 2013)

El uso del estándar IEEE 802.15.4 se ha generalizado ya que tiene un alcance de 10 a 20 metros con un rango de transmisión de datos entre $868 \mathrm{Mhz}: 20 \mathrm{~kb} / \mathrm{s}$ a $2.4 \mathrm{GHz}: 250 \mathrm{~kb} / \mathrm{s}$.

En el caso del protocolo de comunicaciones de IoT se utiliza el de redes de área amplia de baja potencia (Low Power Wide Area Network, LPWAN) ya que permite el envío inalámbrico de datos.

Las empresas que han desarrollado protocolos y proveen servicios en el nivel 2 son diversas $\mathrm{y}$ han mantenido procesos de innovación en el área de IoT (Al-Sarawi, Anbar, Alieyan, \& Alzubaidi, 2017) como:

1. SixFox que utiliza tecnología de banda ultra estrecha (Ultra Narrow Band, UNB);

2. ZigBee para redes de radios digitales de pequeño tamaño y baja potencia que transmiten con transmisión de datos a distancias más largas o

3. RFID: Identificación por radiofrecuencia que si bien incluye una variedad de estándares se caracteriza por habilitar la lectura a distancia.

Las tres tecnologías listadas son las más representativas y se encuentran directamente relacionadas como la tecnología establecida en la capa 3 .

En el nivel 3 se establece el uso del protocolo IPv6 debido a que el incremento de dispositivos obliga a extender el identificador numérico asignado a cada objeto, por lo que si bien su característica principal es el incremento de direccionamiento de 32 bits a 128 bits en esta versión del protocolo también se simplifica el formato de encabezado de los paquetes, se extiende la capacidad para identificar flujos de datos y se mejora la autentificación y la privacidad.
En el caso de las capas 4 a 6, en la tabla 2 se observa la división de estas capas para los protocolos TCP en HTTP, MQTT y REST y para UDP en CoAp y SOAP respectivamente; pero también se tiene la posibilidad de utilizar FTP, SMTP y JMS.

Durante la construcción del proyecto es importante ajustarse a la arquitectura sin que necesariamente deba considerarse como una segmentación explicita de cada uno de los niveles, sino que los componentes empleados se adapten en función de la problemática a resolver y del área de aplicación.

\section{Componentes de un desarrollo de IoT}

A partir de la selección de un modelo de referencia, generalmente esquematizado en capas, se estructuran los proyectos de IoT considerando los componentes mostrados en la Figura 1.

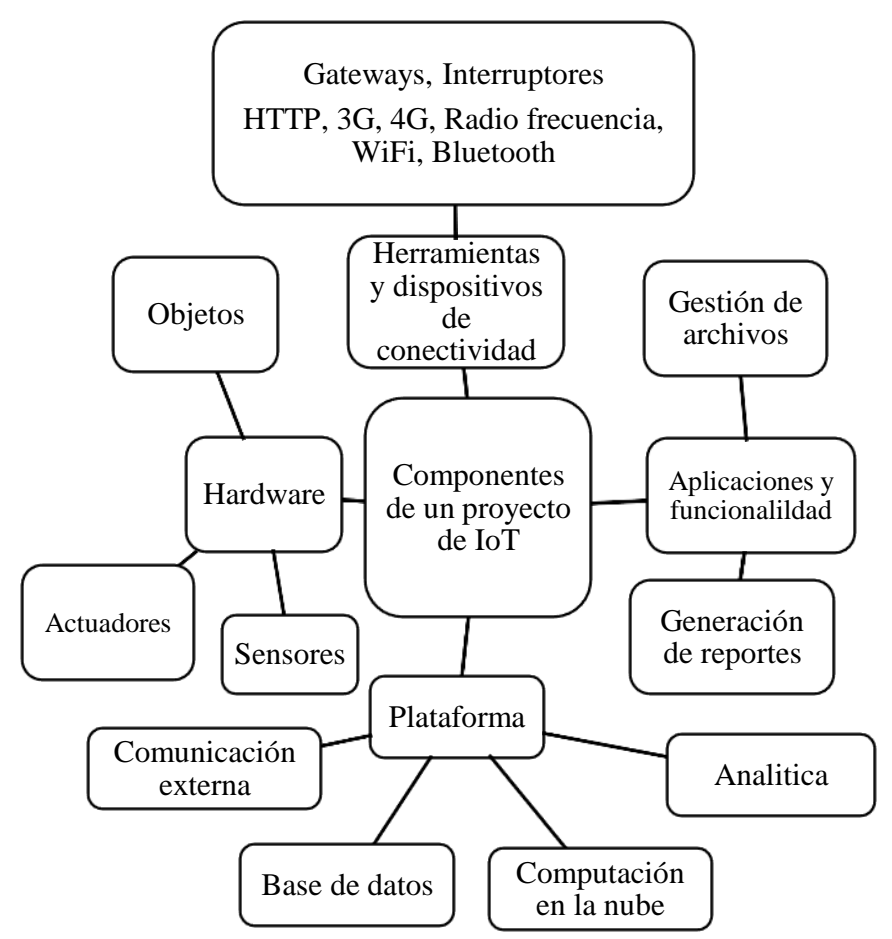

Figura 1 Componentes de un proyecto en IoT Fuente: Elaboración basada en (Bello et al., 2017; Bradley et al., 2014)

Del análisis de la figura 1 se establece que se puede estructurar el proceso de desarrollo comenzando por el hardware o los objetos que envían datos a través de los sensores, mientras los actuadores realizan acciones ya sean periódicas o de ajuste en función del contexto en el que opera la aplicación, y los datos generados viajan empleando las herramientas, los productos y los dispositivos de conectividad. 
La plataforma es indispensable para utilizar los servicios que proporciona el sistema y también se requiere para almacenar la información en la base de datos, puede emplearse de diversos como modos, normalmente identificados como aaS - as a Service (Tabla 3).

\begin{tabular}{|l|l|l|}
\hline Acrónimo & \multicolumn{1}{c|}{ Tipo de servicio } & \multicolumn{1}{c|}{ Descripción } \\
\hline SaaS & Software (SW) & $\begin{array}{l}\text { Aplicaciones ría } \\
\text { disponibles ría } \\
\text { Internet (como } \\
\text { hojas de datos o } \\
\text { compiladores) }\end{array}$ \\
\hline PaaS & Plataforma & $\begin{array}{l}\text { Pila de SW para } \\
\text { uso de diversas } \\
\text { aplicaciones vía } \\
\text { Internet }\end{array}$ \\
\hline IaaS & Infraestructura & $\begin{array}{l}\text { Servidores o discos } \\
\text { de almacenamiento } \\
\text { disponibles a través } \\
\text { de Internet. }\end{array}$ \\
\hline
\end{tabular}

Tabla 3 Relación de servicios proporcionados mediante computación en la nube

En la plataforma también se debe brindar acceso a las aplicaciones de almacenamiento externo de otras compañías con el objetivo de integrar en la aplicación los datos disponibles y los procesos de análisis.

Los componentes identificados en la Figura 1 también permiten establecer los criterios de búsqueda para la especificación de variables que se presentan en el método. Con el objetivo de orientar la investigación se plantean cuatro preguntas centrales de investigación comenzando en la categoría superior de análisis:

¿Cuál es el lenguaje de programación más utilizado para estructurar proyectos de IoT a nivel aplicación?

¿En qué sectores se están desarrollando más aplicaciones de IoT?

¿Cuál es la tecnología de comunicación hacia Internet más empleada?

¿Qué tipos de sensores y actuadores se emplean para la resolución de problemas en los sectores de aplicación de IoT?

\section{Metodología}

La metodología utilizada es cualitativa de alcance descriptivo como los empleados en (Bautista, Parra-Valencia, \& Guerrero, 2017; Ramírez Madrid, D. A., \& Rodríguez Hernández (s/f) y en donde se realizas análisis documentales.
Sin embargo, en este documento el interés está centrado en la identificación de elementos para estructurar de forma ágil proyectos que permitan desarrollar aplicaciones de IoT (o Internet de las Cosas) de acuerdo con la ficha técnica para el estudio descriptivo mostrada en la Tabla 4:

\begin{tabular}{|c|c|c|}
\hline \multicolumn{3}{|c|}{ Ficha técnica del estudio descriptivo } \\
\hline Bases de datos & \multicolumn{2}{|c|}{$\begin{array}{l}\text { Redalyc, SciELO, Dialnet, } \\
\text { RedIB, IEEE, Google académico }\end{array}$} \\
\hline Descriptores & \multicolumn{2}{|c|}{$\begin{array}{l}\text { IoT, Lenguaje de programación, } \\
\text { Plataformas, Aplicaciones, } \\
\text { Elementos de Conectividad }\end{array}$} \\
\hline \multirow[t]{2}{*}{$\begin{array}{l}\text { Criterios de } \\
\text { exclusión e } \\
\text { inclusión }\end{array}$} & Inclusión & $\begin{array}{l}\text { I1: Investigaciones } \\
\text { relacionadas con IoT } \\
\text { en la que se } \\
\text { presentan desarrollos } \\
\text { tecnológicos } \\
\text { I2: Las } \\
\text { investigaciones } \\
\text { presentan elementos } \\
\text { del desarrollo: } \\
\text { lenguaje, plataforma } \\
\text { o conectividad } \\
\end{array}$ \\
\hline & Exclusión & $\begin{array}{l}\text { E1: Se abordan } \\
\text { investigaciones de } \\
\text { IoT pero no se } \\
\text { presentan } \\
\text { desarrollos. } \\
\text { E2: El artículo no } \\
\text { presenta el } \\
\text { documento } \\
\text { completo. } \\
\text { E3: No es un artículo } \\
\text { académico }\end{array}$ \\
\hline $\begin{array}{l}\text { Método de } \\
\text { obtención de } \\
\text { información }\end{array}$ & \multicolumn{2}{|c|}{ Investigación documental } \\
\hline $\begin{array}{l}\text { Procedimiento } \\
\text { de recolección } \\
\text { de datos }\end{array}$ & \multicolumn{2}{|c|}{$\begin{array}{l}\text { Análisis de proyectos } \\
\text { tecnológicos vinculados a IoT }\end{array}$} \\
\hline
\end{tabular}

Tabla 4 Ficha técnica del estudio descriptivo Fuente: Elaboración Propia

Para el análisis de los resultados los descriptores fueron combinados con el elemento central que es IoT o de forma explícita Internet de las Cosas.

\section{Resultados}

De acuerdo con la ficha técnica del estudio descriptivo (Tabla 4) la búsqueda arrojó 734 artículos interrelacionados principalmente entre las bases de datos de IEEE y Google académico, según de los criterios de exclusión fueron descartados 612 por lo que se analizaron 122 artículos. 
Del análisis realizado, en primer lugar se presenta la respuesta de las cuatro preguntas de investigación y en segundo lugar se muestran hallazgos importantes encontrados durante la revisión

(1). En cuanto a los lenguajes de programación empleados para el desarrollo de toda la aplicación de IoT se identifican lenguajes multiplataforma como Java, Python y $\mathrm{C}$ que se encuentran entre los lenguajes más utilizados, así como JavaScript y PHP.

(2). En los sectores con mayor incidencia de desarrollo de aplicaciones de IoT se encuentran el de la salud, el energético y el automotriz. Por lo que existen múltiples oportunidades para el desarrollo de aplicaciones integrales para las ciudades inteligentes (Smart cities) ya que los sectores previamente listados pueden integrarse para mejorar la calidad de vida de los ciudadanos en las megapolis.

(3). Aunque se está extendiendo el uso de la radiofrecuencia la tecnología para la comunicación que se está empleando es el Wi-fi, lo que puede derivarse de la extensión de su utilización, del abaratamiento de hardware para su uso y de la difusión de código abierto para lograr la conexión de elementos a través de este medio.

(4). De los sensores con amplio rango de utilización se encontró que los de proximidad (ultrasónicos), los de temperatura y los de nivel, así como sus diversas combinaciones para mejorar las aplicaciones son los que predominan en utilización.

Aunque los cuatro elementos listados son de los más empleados, también se identificó que JSON es un lenguaje cuyo uso se ha incrementado para la comunicación y el envío de datos a nivel de dispositivos.

Entre las actividades primordiales para la construcción de soluciones de IoT destaca el proceso de comunicación entre objetos que requiere en primera instancia la identificación de un objeto con posibilidades de incorporación de elementos electrónicos y posteriormente lograr su interconexión (Figura 2)

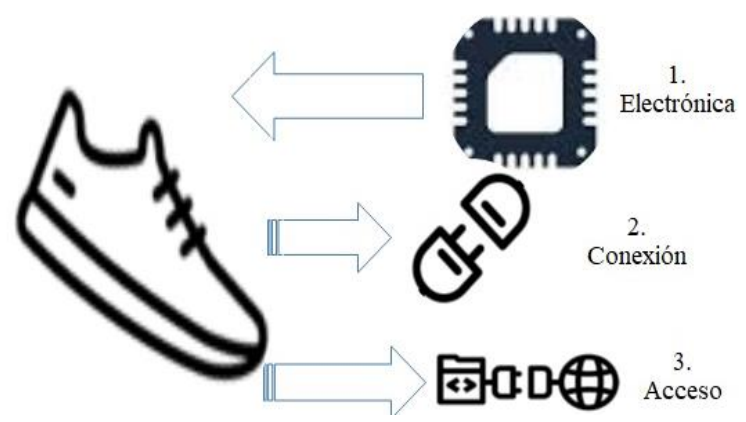

Figura 2 Actividad primaria para el desarrollo de un proyecto en IoT

Fuente: Elaboración Propia

Posterior a la comunicación del objeto es necesario: a) apegarse a una arquitectura por niveles con la abstracción que requiera el desarrollador, b) automatizar todas las operaciones que deban realizarse (como las conexiones a Internet y el uso de una base de datos) para, finalmente c) diseñar la interoperabilidad de la aplicación.

\section{Conclusiones}

La extensión de las aplicaciones de IoT requiere el desarrollo de nuevas habilidades por parte de los programadores, las habilidades están relacionadas con la integración de múltiples componentes, sistemas multiplataformas y herramientas de código abierto.

La seguridad es un tema que adquiere relevancia en el ámbito del Internet de las Cosas debido a la sensibilidad de la información presente en todos los sectores de aplicación, por lo que los esquemas de autentificación son de los elementos cruciales a considerar en el desarrollo de los proyectos.

Lograr la transparencia tecnológica que logre desaparecer de la conciencia de los usuarios el uso de los componentes a través de las aplicaciones constituye uno de los retos del IoT.

Entre las tecnologías primordiales para la construcción de ambientes inteligentes en IoT se encuentran la biotecnología, la nanotecnología y la micro electrónica, así como el desarrollo de materiales avanzados por el que el fomento a estas áreas incrementará el potencial de los proyectos de desarrollo en IoT para la mejora de la calidad de vida de todas las personas. 


\section{Referencias}

Al-Sarawi, S., Anbar, M., Alieyan, K., \& Alzubaidi, M. (2017, May). Internet of Things (IoT) communication protocols. In 2017 8th International conference on information technology (ICIT)(pp. 685-690). IEEE.

Bautista, D. R., Parra-Valencia, J. A., \& Guerrero, C. D. (2017). IOT: Una aproximación desde ciudad inteligente a universidad inteligente. Revista Ingenio Universidad Francisco de Paula Santander Ocaña, 13(1), 920.

Bello, O., Zeadally, S., \& Badra, M. (2017). Network layer inter-operation of Device-toDevice communication technologies in Internet of Things (IoT). Ad Hoc Networks, 57, 52-62.

Bradley, J., Loucks, J., Macaulay, J., \& Noronha, A. Inc. Internet of Everything (IoE) Value Index (White Paper) Cisco Systems. Recuperado de: http://cdn.iotwf.com/resources/71/IoT_Referen ce_Model_White_Paper_June_4_2014.pdf

Evans, D. (2011). Internet de las cosas. Cómo la próxima evolución de Internet lo cambia todo. Cisco Internet Bussiness Solutions Group-IBSG, 11(1), 4-11.

González, D. R. (2013). Arquitectura y Gestión de la IoT. Revista Telemática, 12(3), 49-60.

Gubbi, J., Buyya, R., Marusic, S., \& Palaniswami, M. (2013). Internet of Things (IoT): A vision, architectural elements, and future directions. Future generation computer systems, 29(7), 1645-1660.

Hinden, R. (2017). Internet protocol, version 6 (IPv6) specification.

IOT-A project: disponible en www.iota.eu/public/public-documents/d3.1.

Ramírez Madrid, D. A., \& Rodríguez Hernández, E. D. (s/f). Diseño de un Método para Identificar Necesidades y Oportunidades Para la Implementación de Internet de las Cosas (IoT) Aplicable a Oficinas de Trabajo Donde Permanezcan Entre 30 y 70 Personas y Planteamiento de un Caso Práctico de Solución en las Oficinas de la Agencia Nacional del Espectro.
Rojas, E. (2017). From software-defined to human-defined networking: Challenges and opportunities. IEEE Network, 32(1), 179-185.

Statista. (2018). Internet of Things (IoT) connected devices installed base worldwide from 2015 to 2025 (in billions). Disponible en https://www.statista.com/statistics/471264/iotnumber-of-connected-devices-worldwide/

Tsuzaki, Y., \& Okabe, Y. (2017, January). Reactive configuration updating for IntentBased Networking. In 2017 International Conference on Information Networking (ICOIN) (pp. 97-102). IEEE.

Vermesan O, Friess P, Guillemin P, Gusmeroli S, Sundmaeker H, Bassi A, Jubert IS, Mazura M, Harrison M, Eisenhauer M, et al (2011) Internet of things strategic research roadmap. $\mathrm{O}$ Vermesan, P Friess, P Guillemin, S Gusmeroli, H Sundmaeker, A Bassi, et al, Internet of Things: Global Technological and Societal Trends 1:952 